\title{
Direct Aspiration: Can It Be Aspired?
}

\author{
Jong S. Kim \\ Department of Neurology, Asan Medical Center, University of Ulsan College of Medicine, Seoul, Korea
}

In the recently published trials for endovascular thrombectomy, the procedure was mostly performed with stent retrieval (SR). ${ }^{1,2}$ Direct aspiration (DA) is another method that can be used alone or in combination with SR. Many physicians use DA according to their experiences, but no data are available that support the value of this technique. Given that faster puncture-to-recanalization time is associated with better clinical outcome, DA may be superior to SR. However, DA may result in emboli breaking off distally during withdrawal. Although DA may result in lesser damage to the endothelium than SR, it remains unclear whether this difference is clinically significant.

The two review papers on DA in this issue are timely and interesting. The reviews deserve a separate reading as they discuss this issue in different perspectives. Anderssen and his colleagues ${ }^{3}$ provided a balanced yet critical view on previous trials on DA, such as The Randomized, Concurrent Controlled Trial to Assess the Penumbra System's Safety and Effectiveness in the Treatment of Acute Stroke (THERAPY), Direct Aspiration First Pass Technique for Thrombectomy Revascularization of Large Vessel Occlusion in Acute Ischemic Stroke (ASTER), and their sub-studies, whereas the paper by Kang and Hwang ${ }^{4}$ was more focused on the practical issues and the past and future development of DA techniques. Although the reviews do not give a definite conclusion, they allow us to correctly learn the current status of DA. To solidify evidence, more studies should be performed that compare the safety and efficacy of DA with those of SR. As DA is often used together with $S R$, we may have to compare $S R$ alone with $D A+S R$ as well. Future trials may have to be carefully designed considering that the techniques of DA and SR are continuously developing, and the clinical utility of each technique may differ according to the pathogenesis of stroke (i.e., cardiogenic, artery-to artery embolism, or intrinsic thrombotic occlusion), or the nature and size of the thrombus. Thus, it is not likely that we will obtain a definitive conclusion in the near future. At present, neurologists and interventionists may use DA, SR, or both according to their experiences with consideration of the benefits and harms of these techniques described in the reviews.

Lastly, I am pleased to report that the Journal of Stroke, now in its fifth year, is rapidly growing into a high-standard, internationalized journal. Last year, manuscripts were submitted from more than 50 countries. China was the country that submitted the largest number, followed by South Korea, the United States, Taiwan, Germany, France, Spain, Japan, Australia, the UK, and Italy. Currently, we can accept only about $9 \%$ of them. Our editorial team thanks all the authors and reviewers and will try to publish even higher-quality papers that stroke physicians and researchers can enjoy.

\section{References}

1. Hong KS, Ko SB, Lee JS, Yu KH, Rha JH. Endovascular recanalization therapy in acute ischemic stroke: updated meta-analysis of randomized controlled trials. J Stroke 2015;17:268-281.

2. Yoo $\mathrm{AJ}$, Andersson T. Thrombectomy in acute ischemic stroke: challenges to procedural success. J Stroke 2017;19:121-130.

3. Andersson T, Wiesmann M, Nikoubashman 0 , Gopinathan A, Bhogal P, Yeo LLL. The aspirations of direct aspiration for thrombectomy in ischemic stroke: a critical analysis. J Stroke 2019;21:2-9.

4. Kang $\mathrm{DH}$, Hwang $\mathrm{YH}$. Frontline contact aspiration treatment for emergent large vessel occlusion: a review focused on practical techniques. J Stroke 2019;21:10-22.

\footnotetext{
Correspondence: Jong S. Kim

Department of Neurology, Asan Medical Center, University of Ulsan College of Medicine, 88 Olympic-ro 43-gil, Songpa-gu, Seoul 05505, Korea

Tel: +82-2-3010-3442

Fax: +82-2-474-4691

E-mail: jongskim@amc.seoul.kr
}

The author has no financial conflicts of interest 